\title{
Computational Mechanistic Investigation of Chronotropic Effects on Murine Sinus Node Cells
}

\author{
Benjamin Winkelmann ${ }^{1,2}$, Callum M Zgierski-Johnston ${ }^{1}$, Eike M Wülfers ${ }^{1}$, Jens Timmer ${ }^{2}$, \\ Gunnar Seemann ${ }^{1}$ \\ ${ }^{1}$ Institute for Experimental Cardiovascular Medicine, University Heart Center Freiburg · Bad \\ Kronzingen, Medical Center - University of Freiburg, Germany and Faculty of Medicine, \\ Albert-Ludwigs University of Freiburg, Freiburg, Germany \\ ${ }^{2}$ Physics Institute, Albert-Ludwigs University of Freiburg, Germany
}

\begin{abstract}
It has been shown for multiple species that the beating rate of the sinus node is sensitive to stretch. In rabbit an underlying stretch induced current has been experimentally established. Mathematical models implementing stretch as a non-selective stretch activated current were found to be consistent with the observed increased beating rate. In mice, a negative chronotropic response is observed experimentally. This leads to the question whether non-selective stretch activated currents can also cause a decreased beating rate.

To investigate this, we used a mathematical model of murine sinus node cells and added an ohmic channel. Different levels of stretch were modelled by altering the conductance of the channel.

As we increased stretch in the model, we found an initial decrease in beating rate followed by a monotonic increase, indicating that activation of stretch activated channels can cause opposite chronotropic effects in mice depending on stretch amplitude.
\end{abstract}

\section{Introduction}

The heartbeat is initiated by cardiac pacemaker cells in the sinus node $(\mathrm{SN})$. These cells spontaneously generate action potentials (AP) which spread across the heart. There are pathologies increasing strain in the SN, e.g. a dilated right atrium. In multiple species it has been reported that the beating rate (BR) of the $\mathrm{SN}$ is sensitive to stretch. The effect varies between species, e.g. for rabbit SN an increase in BR upon stretch was observed whereas for mouse the BR decreased [1]. For rabbit SN, it has been shown that the experimentally observed positive chronotropic response is consistent with a mathematical model of single cell rabbit SN. Stretch was modelled as an additional non-selective cation current, caused by stretch activated channels (SAC).

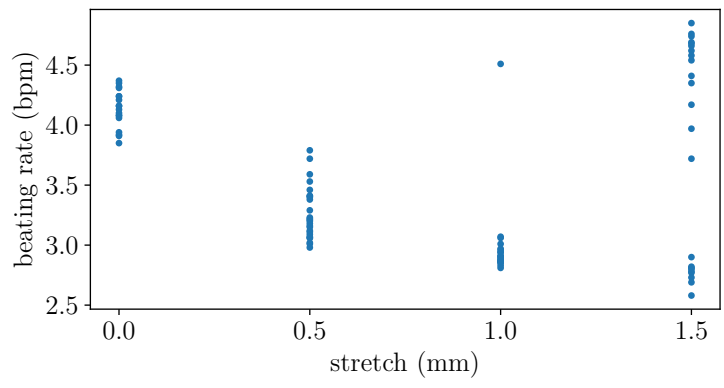

Figure 1. Experimental data of the BR of a murine SN when stretched. Data points in blue, averages in orange.

Patch clamp experiments on longitudinal stretched cells confirmed the presence of such a current [2]. In both model and experiment, the change in BR was accompanied by a reduction in absolute amplitudes of both maximum systolic and maximum diastolic potentials (MSP and MDP, respectively).

Experiments on murine $\mathrm{SN}$ instead showed a negative chronotropic response ([1], fig. 1), raising the question whether SAC activation can also explain the slowed BR upon SN stretch in the mouse.

\section{Methods}

All animal experiments were carried out according to the guidelines stated in the Directive 2010/63/EU of the European Parliament on the protection of animals used for scientific purposes and were approved by the local authorities in Baden-Württemberg, Germany.

A 17-week old mouse was euthanised by cervical dislocation, its chest opened, heart removed and placed in heparin-containing (10 units $/ \mathrm{mL})$ Tyrode solution. The Tyrode solution contained [in $\mathrm{mM}$ ]: $140 \mathrm{NaCl}, 6 \mathrm{KCl}$, $1 \mathrm{MgCl}_{2}, 1.8 \mathrm{CaCl}_{2}, 10$ glucose and 10 HEPES, pH corrected to 7.4 using $\mathrm{NaOH}$. The $\mathrm{SN}$ was then isolated and clamped in a vertical position in a bath filled with oxy- 
genated, room temperature Tyrode solution. The top clamp connected to a force transducer (FORT10, WPI, FL, USA) and the bottom to a micromanipulator. Once mounted, the micromanipulator was adjusted to the point at which diastolic force began to increase, at which point the preparation was shortened to the point at which no diastolic force was measured (about $8 \mathrm{~mm}$ length). The BR was measured between 18 and 25 times at $0 \mathrm{~mm}, 0.5 \mathrm{~mm}, 1.0 \mathrm{~mm}$ and $1.5 \mathrm{~mm}$ stretch with a measurement approximately every minute. Length was increased sequentially. The power spectral density of the force signal over 10 beats was found and the first non-zero peak was the BR.

We used a mathematical model for SN cells of mice [3]

$$
\begin{gathered}
\frac{d V_{m}}{d t}=-\frac{1}{C_{m}} I_{m}\left(V_{m}, \boldsymbol{u}\right) \\
\frac{d \boldsymbol{u}}{d t}=f\left(V_{m}, \boldsymbol{u}\right)
\end{gathered}
$$

where $V_{m}$ is the transmembrane voltage, $C_{m}$ is the capacitance of the cell membrane, $I_{m}$ is the transmembrane current and $\boldsymbol{u}$ is a vector of gating variables and ion concentrations. To integrate non-selective SAC into this model, we added a current

$$
I=g \cdot\left(V_{m}-E\right)
$$

where $g$ is the conductance and $E$ the reversal potential of the SAC. To model the effect of different levels of stretch, $g$ was varied for each simulation in steps of $1 \mathrm{pS} / \mathrm{pF}$ beginning from 0 to $20 \mathrm{pS} / \mathrm{pF}$ and then in steps of $2 \mathrm{pS} / \mathrm{pF}$ up to $48 \mathrm{pS} / \mathrm{pF}$. We calculated $V_{m}$ over a time interval of $5 \mathrm{~s}$ by numerically solving the ODE with a forward Euler scheme (stepsize $10^{-6} \mathrm{~s}$ ).

The first $3 \mathrm{~s}$ were discarded as transient. The cycle length $T$ was determined by averaging peak to peak time intervals. The reversal potential of non-selective SAC is expected to be between 0 and $-20 \mathrm{mV}$ [2]. Therefore we calculated $T$ also for $E=0,-10 \mathrm{mV}$ and $-20 \mathrm{mV}$.

To check whether the ability of the SN to excite surrounding atrial tissue is affected with changed SAC conductance, we used a one dimensional tissue model and the monodomain approach:

$$
\frac{\partial V_{m}}{\partial t}=\frac{1}{C_{m}}\left(\frac{\partial}{\partial x} D \frac{\partial V_{m}}{\partial x}-I_{m}\right)
$$

where $D$ is a conduction parameter adjusting the conduction velocity $(\mathrm{CV})$. The model consists of a central SN strand (red in fig. 2) using the described cell model [3]. This strand has a length of $0.7 \mathrm{~mm}$. The model is extended symmetrically on both ends of the center by a strand of peripheral SN cells of length $0.2 \mathrm{~mm}$ (green). For these cells, Calcium conductances $g_{C a L, 1.2}$ and $g_{C a L, 1.3}$ were increased by a factor 11.37 , the same ratio as in the $\mathrm{SN}$

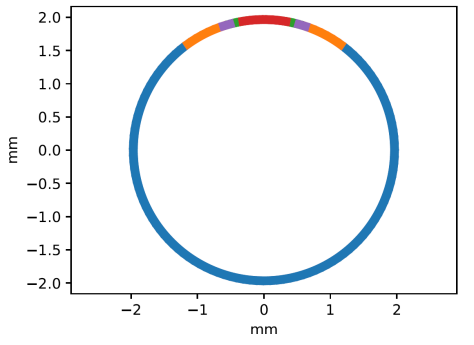

Figure 2. One dimensional ring model of murine SN and atrial tissue. Red marks the central SN. Green are peripheral SN cells, using a modified cell model. Purple marks the junction between $\mathrm{SN}$ and atrial tissue. In orange, a region of outward increasing conductivity is represented. Blue represents atrial tissue.

Table 1. Conduction parameter $D$ and cell model for different tissue of the 1-d model. *In peripheral SN cells, the Calcium conductance was increased by a factor of 11.37 . **For atrial cell models, the parameters $\tau_{d}$ and $\tau_{r}$ were multiplied by a factor of 0.5182 and 7.0238 , respectively.

\begin{tabular}{lll}
\hline Type & $D / D_{\text {centrals }}$ & cell model \\
central SN & 1 & Kharche et al.[3] \\
peripheral SN & 5.0 & Kharche et al. ${ }^{*}[3]$ \\
SN-atria junction & 2.9 & - \\
connective tissue & $14.7-36.7$ & Inada et al. ${ }^{* *}[5]$ \\
atrial tissue & 36.7 & Inada et al. $^{* *}[5]$ \\
\hline
\end{tabular}

model by Zhang et al. [4]. $D$ was chosen such that the CV within the SN was $60 \mathrm{~mm} / \mathrm{s}$ (matching [4]). The peripheral $\mathrm{SN}$ is coupled via a junction (purple) to atrial tissue. Here, the cell model by Inada et al. [5] was used, with parameters $\tau_{d}$ and $\tau_{r}$ multiplied by 0.5182 and 7.0238 , respectively, to match mouse atrial AP durations. The atrial strand had a length of $5.6 \mathrm{~mm}$. The conductivity increases linearly outward over a length of $0.6 \mathrm{~mm}$ (orange) from the value in the $S N$ to a maximum value [6]. This value was adjusted to set the $\mathrm{CV}$ to $500 \mathrm{~mm} / \mathrm{s}$. To avoid boundary effects, the model was structured as a ring. The values for the conduction parameter can be found in tab. 1 .

\section{Results}

The experimental results of BR change depending on stretch are shown in fig. 1 . For $0.5 \mathrm{~mm}$ and $1 \mathrm{~mm}$ stretch lead to a negative chronoropic response, as the average BR decreased by $21 \%$ and $28 \%$ respectively. For $1.5 \mathrm{~mm}$ stretch the response was split into two clusters, one negative and one positive chronotropic.

An excerpt of $V_{m}$ progression for no additional channel $(g=0), g=12 \mathrm{pS} / \mathrm{pF}$ and $g=32 \mathrm{pS} / \mathrm{pF}$ with $E=0$ as calculated with the single cell model is shown in fig. 3 . With increasing the conductance of the SAC, the maxi- 


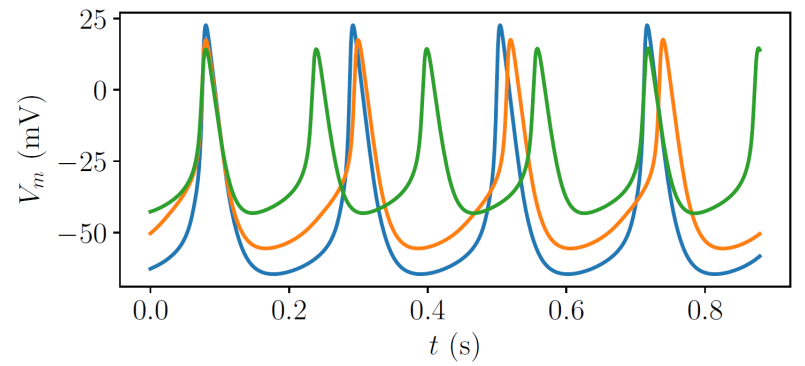

Figure 3. $V_{m}$ - $t$ diagram for reversal potential $E=0$ and conductances $g=0$ (blue), $g=12 \mathrm{pS} / \mathrm{pF}$ (orange) and $g=32 \mathrm{pS} / \mathrm{pF}$ (green).

mum diastolic potential (MDP) was shifted to more positive values; the voltage amplitude and maximum upstroke velocity were reduced (tab. 2).

The relationship between conductance $g$ and the frequency $f$ of AP is shown in fig. 4. For the three reversal potentials $E=0 \mathrm{mV},-10 \mathrm{mV},-20 \mathrm{mV}$, the frequency increased marginally $(<1 \%$ ) for small $g$. As $g$ was increased further, the frequency decreased until a local minimum was reached, followed by a monotonous increase. More negative reversal potentials shifted this minimum towards higher conductances and decreased the minimum frequency (tab. 3). The results of the tissue model (dashed lines in fig. 4) exhibit a similar tri-phasic behaviour as the single cell model. The base frequency is lower compared to the single cell case and the minima are more pronounced, both in absolute and relative scale. For more negative reversal potentials, the $\mathrm{SN}$ loses ability to pace the atrium at lower conductances $g$.

Table 2. MDP, MSP, voltage amplitude $(\Delta V)$ and maximum upstroke velocity $\left(d V / d t_{\max }\right)$ for different conductances of the SAC with reversal potential $E=0$.

\begin{tabular}{lllll}
\hline \hline$g$ & MDP & MSP & $\Delta V$ & $\begin{array}{l}d V / d t_{\max } \\
\mathrm{pS} / \mathrm{pF}\end{array}$ \\
$\mathrm{mV}$ & $\mathrm{mV}$ & $\mathrm{mV}$ & $\mathrm{V} / \mathrm{s}$ \\
\hline 0 & -64.5 & 22.6 & 87.1 & 9.2 \\
12 & -55.6 & 17.7 & 73.3 & 6.3 \\
32 & -43.3 & 15.0 & 58.3 & 5.3 \\
\hline \hline
\end{tabular}

Table 3. Location of the frequency minimum (fig. 4) for different E. Values for the 1-d tissue model in parenthesis.

\begin{tabular}{llll}
\hline \hline$E$ & $g_{\min }$ & $f_{\min }$ & $f_{\min } / f_{0}$ \\
$\mathrm{mV}$ & $\mathrm{pS} / \mathrm{pF}$ & $\mathrm{Hz}$ & \\
\hline 0 & $11.1(10.6)$ & $4.53(4.26)$ & $96 \%(94 \%)$ \\
-10 & $13.3(12.5)$ & $4.40(4.12)$ & $93 \%(91 \%)$ \\
-20 & $16.2(15.0)$ & $4.18(3.87)$ & $89 \%(85 \%)$ \\
\hline \hline
\end{tabular}
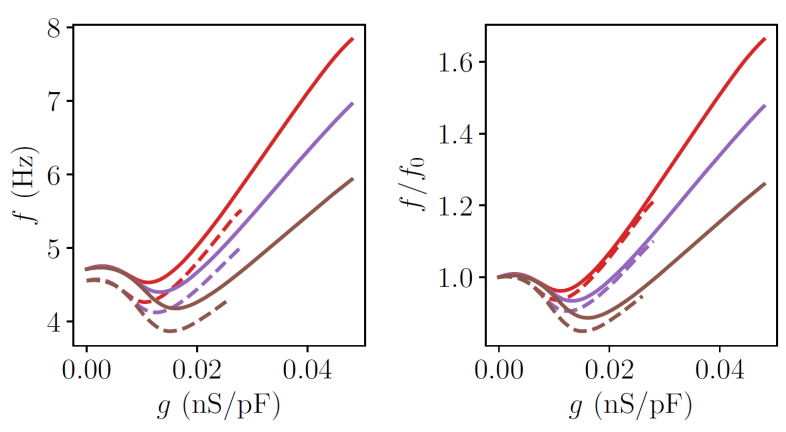

Figure 4. The frequency of the AP plotted against the conductance of the SAC (left). On relative scale normalized with the value for $g=0$ (right). Different colours correspond to different reversal potentials $E=0$ (red), $E=-10 \mathrm{mV}$ (purple), $E=-20 \mathrm{mV}$ (brown). The dashed lines indicate results of tissue simulations in a 1$\mathrm{d}$ model. Dashed lines end at conductances, for which the SN did not activate the tissue anymore.

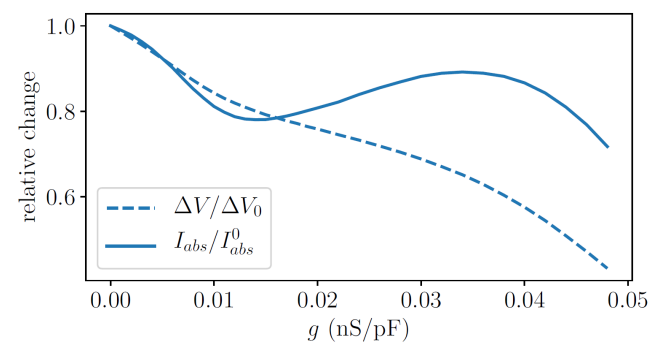

Figure 5. Relative changes of the amplitude $\Delta V$ and the mean absolute current $\bar{I}_{a b s}$ as $g$ is increased.

\section{Discussion}

We define the mean absolute current $\bar{I}_{a b s}-$ the absolute current flowing through the membrane averaged over one cycle - as

$$
\bar{I}_{a b s}:=\frac{1}{T} \int_{t_{0}}^{t_{0}+T}\left|I_{m}\right| d t
$$

where $T$ is the basic cycle length. For the single cell model using equation 1 we can relate the frequency $f=1 / T$ to $\bar{I}_{a b s}$ and the voltage amplitude $\Delta V$

$$
f=\frac{1}{2 C_{m}} \frac{\bar{I}_{a b s}}{\Delta V}
$$

From eq. 6 we know for the single cell model a change in frequency has to coincide with a change in mean absolute current and/or a change in amplitude. As seen in fig. 5, the amplitude decreased with increased SAC conductance, which would increase the frequency as a direct result. However, the reshaping of the voltage curve affects the currents flowing through the membrane. Together with the added SAC current, the mean absolute current decreases for low conductances. The chronotropic response 


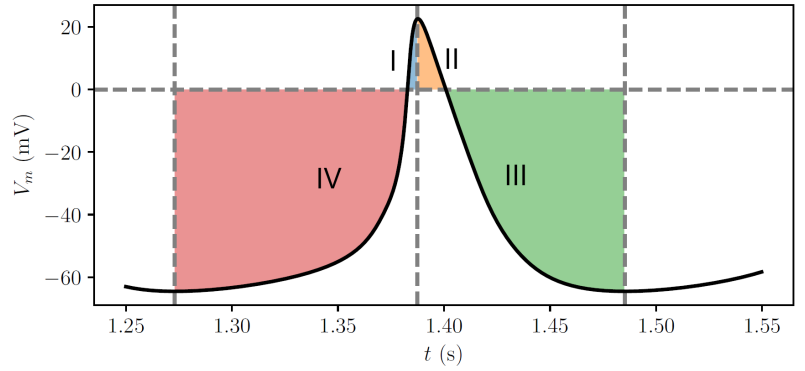

Figure 6. AP for $g=0$. The coloured areas mark phases during which SAC with $E=0$ would decelerate depolarisation (I), accelerate re-polarisation (II), decelerate repolarisation (III) and accelerate depolarisation (IV).

is negative if the relative decrease in $\bar{I}_{a b s}$ exceeds the relative decrease in amplitude (solid line below dashed line in fig. 5). In a way it is surprising that the addition of SAC actually decreased $\bar{I}_{a b s}$ : Refering to fig. 6, one can see that the AP is dominated by phases during which the current of SAC with reversal potential $E=0$ would increase $\bar{I}_{a b s}$ (phases II and IV). This means the negative chronotropic response is a result of the coupling of SAC via the voltage to other ion channels (fig. 7), leading to an overall decreased mean absolute current, exceeding the (relative) decrease in amplitude.

In the tissue model, the tri-phasic pattern of the chronotropic response was sustained. This may be a consequence of the low conductance within the $\mathrm{SN}$ and the $\mathrm{SN}$-atria junction, as the $\mathrm{SN}$ cells remain relatively isolated and therefore the voltage dynamic remains mostly driven by the transmembrane currents. The inability to excite tissue at higher SAC conductances can be explained by the reduced maximum voltage. The SAC current "pulls" $V_{m}$ towards its reversal potential, reducing the magnitude of MSP and MDP and thereby diminishing the maximal voltage gradient between atria and SN. Thus, the current from SN to atria is reduced until the current is too weak to depolarise atrial cells beyond threshold. For more negative reversal potentials, the maximum voltage is reduced stronger for the same $g$, or in other words the same reduction in maximum voltage occurs at lower SAC conductances. This explains why SN pacing is lost at lower conductances for more negative reversal potentials.

Quantitatively, the negative chronotropic responses of the model (at most $15 \%$ for $E=-20 \mathrm{mV}$ in the 1-d tissue) lie within the range of responses as experimentally observed in mice [1]. A switch from negative to positive chronotropic response has not been reported in literature and our experimental data is not conclusive (only one subject and diverse response at high stretch). Still, our simulations indicate that activation of SAC can cause a negative chronotropic response in murine $\mathrm{SN}$ - the opposite response they have in rabbit.
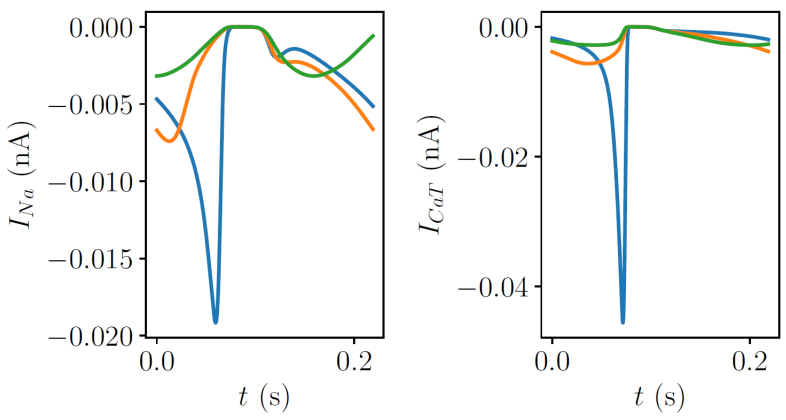

Figure 7. Sodium current $I_{N a}$ (left) and Calcium current $I_{C a T}$ (right) over one cycle, for different conductances of the SAC: $g=0$ (blue), $g=12 \mathrm{pS} / \mathrm{pF}$ (orange), $g=32 \mathrm{pS} / \mathrm{pF}$ (green).

\section{Acknowledgements}

We thank Dr. S. Kharche for providing us with the original source code of the model.

\section{References}

[1] Cooper PJ, Kohl P. Species- and preparation-dependence of stretch effects on sino-atrial node pacemaking. Annals of the New York Academy of Sciences 2005; 1047:324-335.

[2] Cooper PJ, Lei M, Cheng L, Kohl P. Selected Contribution: Axial stretch increases spontaneous pacemaker activity in rabbit isolated sinoatrial node cells. Journal of Applied Physiology 2000; 89:5:2099-2104.

[3] Kharche S, Yu J, Lei M, Zhang H. A mathematical model of action potentials of mouse sinoatrial node cells with molecular bases. American Journal of Physiology-Heart and Circulatory Physiology 2011; 301:3:H945-H963.

[4] Zhang H, Holden AV, Kodama I, Honjo H, Lei M, Varghese T, Boyett MR. Mathematical models of action potentials in the periphery and center of the rabbit sinoatrial node. American Journal of Physiology-Heart and Circulatory Physiology 2000; 279:H397-H421.

[5] Inada S, Hancox JC, Zhang H, Boyett MR. Onedimensional mathematical model of the atrioventricular node Including atrio-nodal, nodal, and nodal-his cells. Biophysical Journal 2009; 97:2117-2127

[6] Joyner RW, van Capelle FJ. Propagation through electrically coupled cells. How a small SA node drives a large atrium. Biophysical Journal. 1986;50(6):1157-1164.

Address for correspondence:

Gunnar Semann

Elsässer Straße 2q, 79110 Freiburg

gunnar.seemann@universitaets-herzzentrum.de 